\title{
Kinematics and morphology of warped disk galaxies
}

\author{
Gyula Józsa* \\ RAIUB, Bonn, Germany \\ E-mail: gjozsalastro.uni-bonn.de
}

\section{Tom Oosterloo}

ASTRON, Dwingeloo, The Netherlands

E-mail: oosterloodastron.nl

\section{Uli Klein}

RAIUB, Bonn, Germany

E-mail: ukleineastro.uni-bonn.de

\section{Franz Kenn}

RAIUB, Bonn, Germany

E-mail: fkenn@astro.uni-bonn.de

\begin{abstract}
Theories that explain warps in disks of galaxies usually make an interaction with their environment responsible for their appearance. According to a large number of these theories, the formation of a warp is caused by a misalignment of a triaxial dark matter halo with the disk. This misalignment is maintained or frequently excited by cosmic infall or gravitational interaction with companions. Such a misalignment should cause measurable signatures in the kinematics of the disk material, which are expected to be largest for extreme warps. We present deep HI synthesis observations and complementary photometric observations of five disk galaxies, three of which show extreme warps. We discuss the first results of a tilted-ring analysis of the galaxies' gaseous disks.
\end{abstract}

Baryons in Dark Matter Halos

5-9 October 2004

cat /dev Novigrad, Croatia

${ }^{*}$ Speaker. 


\section{Introduction}

Scenarios for warp formation in disk galaxies may be divided into two classes. Those which use the gravitational impact of the dark matter halo as being involved in the warp formation, those which use interaction with the intergalactic medium, the gas (e.g. López-Corredoira et al. 2002, Sánchez-Salcedo 2004) or the magnetic fi eld (e.g. Battaner et al. 1990), as an explanation for the formation of warps. Scenarios belonging to the fi rst class generally make a misalignment of a non-spherical halo with the galaxy disk responsible for the warping. The resulting torque on the disk excites the warp. As an explanation for the longevity or the frequent excitation of the misalignment, which is necessary to explain the frequency of warps, either cosmic infall (e.g. Jiang \& Binney 1999, Bailin \& Steinmetz 2002) or tidal interaction with a companion are made responsible (Weinberg 1998, Bailin 2003). In order to test this misalignment hypothesis, we are performing a detailed study of warps in 5 selected galaxies known to possess regular and symmetric large-scale warps. If warps are due to a misalignment between the disk and the non-spherical halo, the force fi eld acting on the gas and the stars is not spherically symmetric and changes with the warp orientation. These changes should be strongest in galaxies with strong warps. Here, we present the observational data, HI spectroscopy obtained with the WSRT and optical (Sloan Gunn $i^{\prime}$ band) photometry obtained with the INT. We show our fi rst results parametrising the galaxies by means of a least-squares fi $t$ of a tilted-ring model to the data cubes.

\section{Observations and data reduction}

The most suitable objects for our project are galaxies with an extremely warped HI disk that show both regular kinematics and morphology, i.e., they should be symmetric with respect to the centre. As objects already known to have these properties are rare, we put no further constraints on the galaxies of our small sample. It contains three galaxies with extreme warps (NGC 2685, NGC 3718, NGC 5204) and two with moderate warps (UGC 3580, NGC 2541).

The HI observations took place between December 2002 and May 2003. The data were reduced with the Miriad reduction software package. Depending on the observing time we reached a minimum noise level of 0.24 to $0.5 \mathrm{mJy} /$ beam and a minimum HPBW of 12 ". The optical observations were performed in February/March 2004 under varying weather conditions during grey/bright time. Four out of seven granted nights proved to deliver useful data over large periods of time. We observed each object of the sample in a series of subsequent short exposures with a maximum of $400 \mathrm{~s}$ exposure time. To obtain a co-added image from the data we made use of the GaBoDS Wide Field Imager reduction pipeline (Schirmer et al. 2003). The fi nal images have a $\sigma_{\text {rms }}$ between 25 and $26 \mathrm{mag}$ in an effective area of $1 \operatorname{arcsec}^{2}$.

\section{The tilted-ring model}

As a fi rst approach we parametrise the HI kinematics of the observed galaxies by means of the tilted-ring model (Rogstad et al. 1974). A galactic disk is represented by a number of concentric and mutually inclined rotating rings, which are described by a set of parameters the most important of which are a) the (common) central position and the systemic velocity, b) the face-on surface 
density, c) the rotation velocity, d) the inclination and the position angle describing the spatial orientation of each ring.

The methods currently used to fi t a tilted-ring model to observational data are somewhat restricted. The usually adopted method is to fi t to the velocity fi eld (Begemann 1987), which is bound to fail if the line-of-sight crosses the disk more than once, as it is the case for some of our sample galaxies. We therefore developed an automated routine based on currently available software (Miriad, GIPSY) that allows a direct fi t of a tilted-ring model to a data cube. For a set of parameters, a model data cube is computed by Monte-Carlo integration and subsequent convolution with an artifi cial observing beam. The quality of such a model is determined by the comparison with the original data cube evaluating the quadratic sum of the difference $\chi^{2}$ between the original and modelled data cube. In our fitting process we minimize the $\chi^{2}$ by a cyclic optimization of each single parameter in the model. While our method is expensive in terms of computing-time and thus does not (yet) allow the calculation of statistical errors, we reach the regime of a well-defi ned minimum in parameter-space and obtain very reliable models. The strongest source for disagreements between model and the measurement are the (unavoidable) deviations of the galaxy morphology from the assumed symmetry inherent to the tilted-ring model. Except for the interacting galaxy NGC 3718, signifi cant deviations are visible only in the outermost regions of the sample galaxies at the $\leq 3 \sigma_{\text {rms }}$ level.

\section{Preliminary results and outlook}

The geometry of the projected disks of the sample galaxies is the same in the stellar- and the HI component out to the optical detection limits. It is therefore unlikely that interaction with gas or magnetic fi elds outside the galaxies causes the warping in these systems, since a deviation of the optical and gaseous disks would be expected. We could not find previously unknown companions to our sample galaxies, with the possible exception of NGC 2685. However, a possible LSB companion to this galaxy seen in the optical has no pronounced HI counterpart and might be a background galaxy. Except for NGC 3718 none of our sample galaxies shows any sign of tidal interaction with a companion, such as tidal streams.

Most of our sample galaxies are chosen to be extreme cases of warped galaxies and thus having distinct morphologies and kinematics, albeit the fact that they proved to maintain the desired symmetry even when pushing down the detection limits. An exception is the interacting galaxy NGC 3718. Nevertheless from our parametrisations we can report tendencies that are common to all galaxies in our sample:

1. Our best-fi t models never show a flat inner part, including the moderate cases. Given the fact that we currently have a poor handle on the statistical errors, a flat inner disk is possibly consistent with our models of NGC 2541, UGC 3580, and NGC 5204. Nevertheless, it is feasible to determine a region within which the disk geometry changes substantially, thus marking the start of an outer warp, comparable to former fi ndings for "normal" disk galaxies (Briggs 1990).

2. The outer warp starts where the surface density reaches a constant low level. This may be interpreted as an at least equally important addendum to the rule that a warp starts at the edge of the optical disk (Briggs 1990): It also starts at the "edge" of the bright HI disk.

3. We detect a change of the rotation velocity with the orientation of the disk. The most pronounced 
cases are NGC 2685 and NGC 5204. First attempts to reconstruct the measured data cube with a constant rotation velocity after the turn-over radii failed. This means that in these two (extreme) cases we detect the change of the net gravitational force-fi eld with the orientation of the disk, while the same tendency is seen for all of our sample galaxies.

4. As it has already been claimed as a general rule for the behaviour of warps by Briggs (1990) on the basis of the orientation of the line-of-nodes, the geometry of our sample galaxies shows two different coherent regimes that adjoin at the commencement of the outer warp. Except for the case of UGC 3580, the change of the tilt of the disk with itself in the outer regime is much less pronounced than in the inner one. This points to the fact that a warp marks a transition between two distinct dynamical systems. We stress the fact that this fi nding takes the whole disk geometry into account and not only the orientation of the line-of-node. It is therefore completely independent of the orientation of the galaxies in the sky.

While our fi tting method is very successful in the shown cases and results in new insights into the kinematics of warps, its drawbacks are obvious. The procedure is as yet too slow to determine statistical errors, and the motion of the disk material is assumed to be circular, while of course orbits of the disk material in a non-spherical potential are most likely to be circular only to zeroth order. We are well on the way with developing an optimised, faster computer code that additionally will allow for velocity variations along and perpendicular to the rings, enabling us to confi rm our results and to perform a kinematic analysis beyond the tilted-ring model.

An analysis of the light distribution will allow us to put constraints on the gravitational potential of the stellar and gaseous disk, and hence on the threedimensional structure of the DM halo.

\section{References}

[1] Bailin, J., Steinmetz, M., 2003, Ap\&SS, 284, 701

[2] Bailin, J., 2003, ApJ, 583, 79

[3] Battaner, E., Florido, E., Sanchez-Saavedra, M. L., 1990, A\&A 236, 1

[4] Begemann, K.G., 1987, PhD Thesis, Groningen

[5] Briggs, F.H., 1990, ApJ, 352, 15

[6] Jiang, I., Binney, J., 1999, MNRAS 303, 7

[7] López-Corredoira, M., Betancort-Rijo, J., Beckman, J.E., 2002, A\&A 386, 169

[8] Rogstad, D.H.; Lockart, I.A.; Wright, M.C.H., 1974, ApJ, 193, 309

[9] Sánchez-Salchedo, 2004, poster shown at "The environments of galaxies: From kiloparsecs to megaparsecs", Swinburne University

[10] Schirmer, M., Erben, T., Schneider, P., Pietrzynski, G., Gieren, W., Carpano, S., Micol, A., Pierfederici, F., 2003, A\&A 407, 869

[11] Weinberg, M.D., 1998, MNRAS 299, 273

Acknowledgments: We thank Thomas Erben for providing the brilliant WFI reduction pipeline and his aid reducing the optical data.

[h] 


\begin{tabular}{|r|r|r|r|r|r|}
\hline Name: & NGC 2541 & NGC 2685 & UGC 3580 & NGC 3718 & NGC 5204 \\
\hline Type: & SA(s)cd & (R)S0+pec & SA(s)a pec & SB(s)+pec & SA(s)m \\
Total B-magnitude: & $-18.5 \mathrm{mag}$ & $-18.6 \mathrm{mag}$ & $-18.2 \mathrm{mag}$ & $-20 \mathrm{mag}$ & $-17.7 \mathrm{mag}$ \\
Distance: & $7.4 \mathrm{Mpc}$ & $13.3 \mathrm{Mpc}$ & $16 \mathrm{Mpc}$ & $15.6 \mathrm{Mpc}$ & $2.7 \mathrm{Mpc}$ \\
Optical diameter: & $378^{\prime \prime} \widehat{=} 13.6 \mathrm{kpc}$ & $301^{\prime \prime} \widehat{=} 19.5 \mathrm{kpc}$ & $204^{\prime \prime} \widehat{=} 15.8 \mathrm{kpc}$ & $448^{\prime \prime} \widehat{=} 34 \mathrm{kpc}$ & $300^{\prime \prime} \widehat{=} 4 \mathrm{kpc}$ \\
HI diameter: & $780^{\prime \prime} \widehat{=} 28 \mathrm{kpc}$ & $610^{\prime \prime} \widehat{=} 39 \mathrm{kpc}$ & $520^{\prime \prime} \widehat{=} 40.3 \mathrm{kpc}$ & $980^{\prime \prime} \widehat{=} 74 \mathrm{kpc}$ & $910^{\prime \prime} \widehat{=} 12 \mathrm{kpc}$ \\
\hline
\end{tabular}

Table 1: Basic properties of the galaxy sample

Figs. 1-5: Our observations and illustrations of our best-fi t models: a) An overlay of the HI total intensity maps (contours) on the optical i' band images (greyscale). b) An overlay of a projection of fi tted rings (coloured ellipses) on the HI total intensity map (greyscale) along with the line-ofnodes from our models (solid line). c) A "Tiltogram" showing the tilt angle of the modelled disk with itself at two radii. The axis units are arcsec. d) An overlay of the HI total intensity maps of our observations on the total intensity maps from our models. The solid line marks the direction along which the position-velocity diagrams e) and f) are carried out. e) Position-velocity diagram of the models carried out along the solid line in d). f) Position-velocity diagram of our observations carried out along the solid line in d).

a)
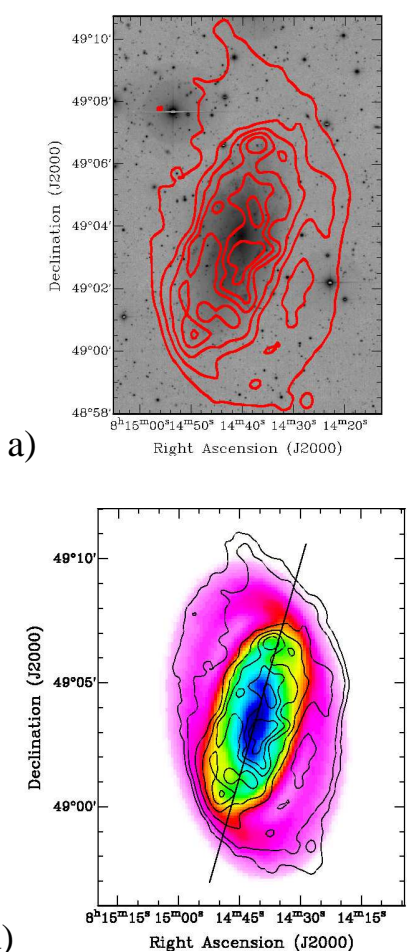

b)
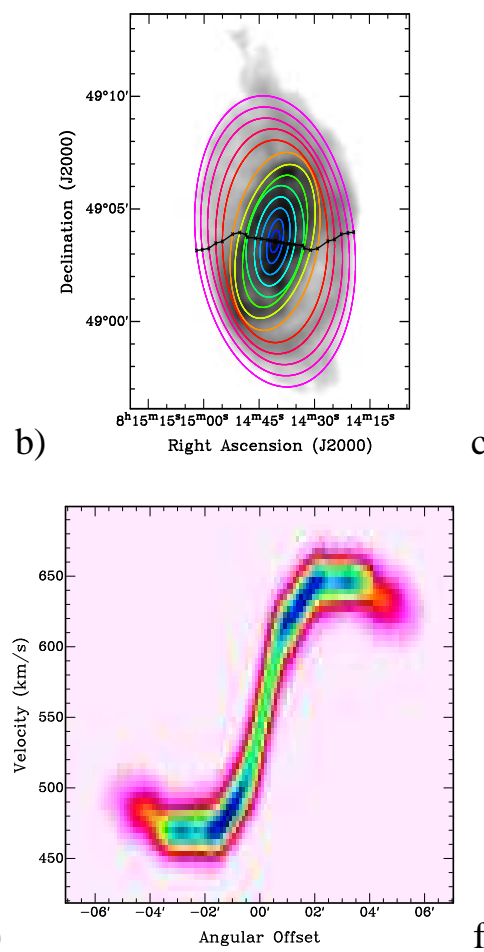

c)
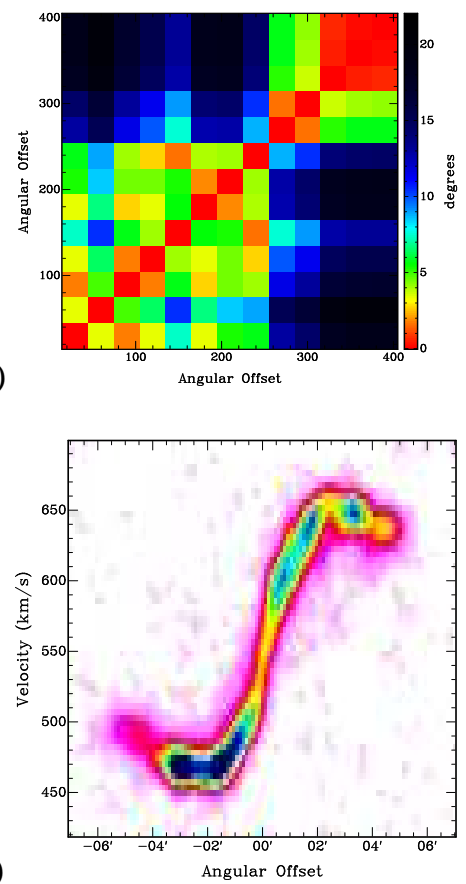

Figure 1: NGC 2541, contours in a) $2,5,8,11,14,17 \mathrm{M}_{\odot} / \mathrm{pc}^{2}$, in d) $0.5,1,2,5,8,11,14,17 \mathrm{M}_{\odot} / \mathrm{pc}^{2}$ 
a)

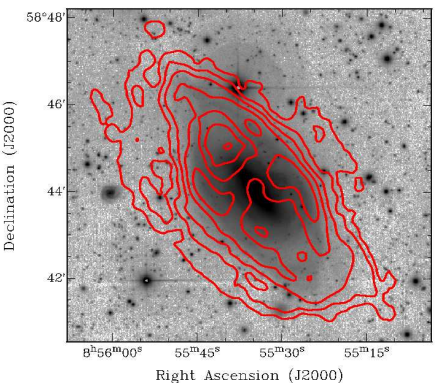

d)

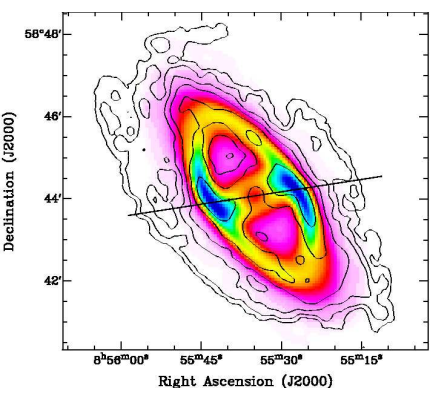

b)
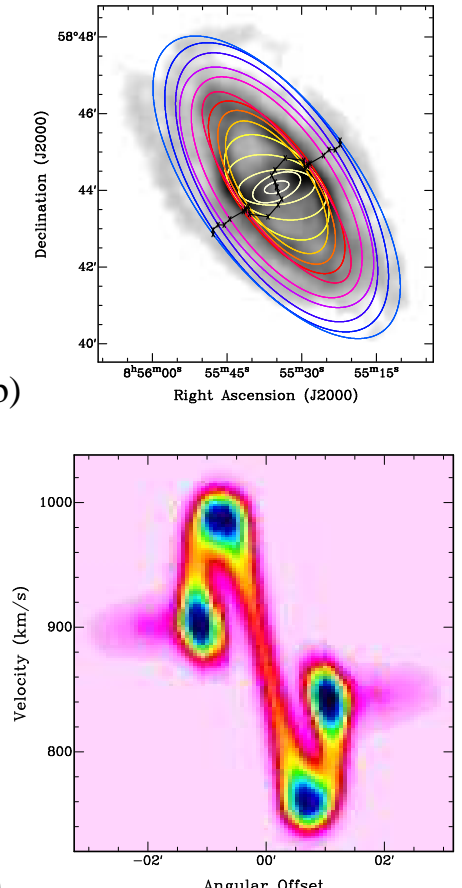

c)

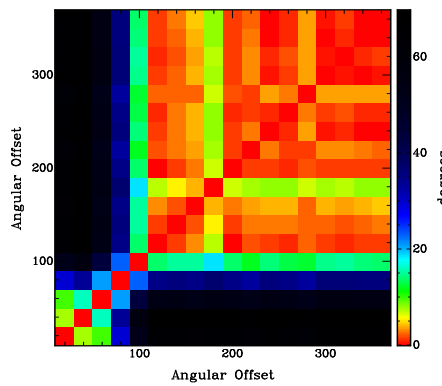

f)

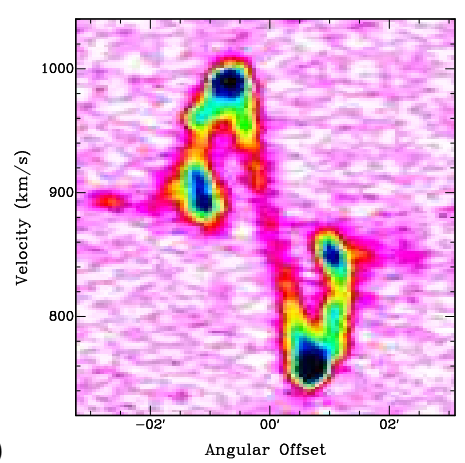

Figure 2: NGC 2685, contours in a) $0.6,1.2,2.4,4.8,9.6 \mathrm{M}_{\odot} / \mathrm{pc}^{2}$, in d) $0.3,0.6,1.2,2.4,4.8,9.6 \mathrm{M}_{\odot} / \mathrm{pc}^{2}$

a)
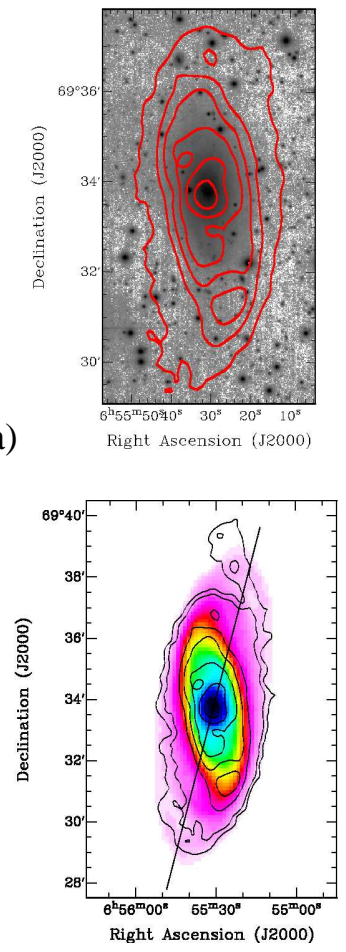

b)

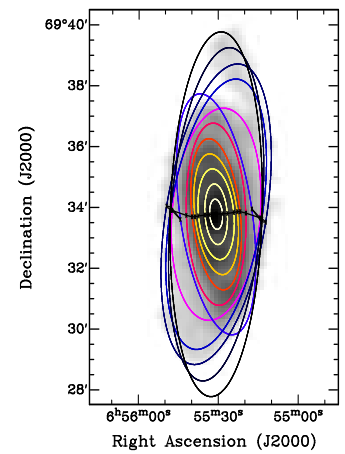

e)

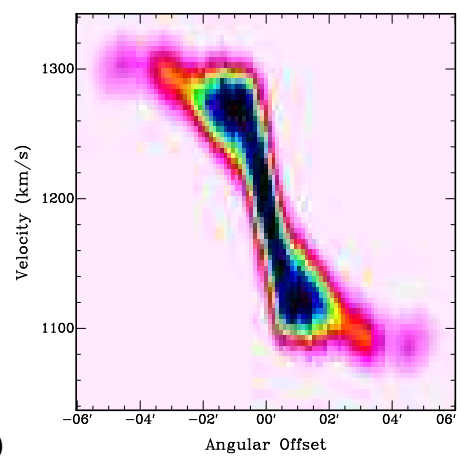

c)
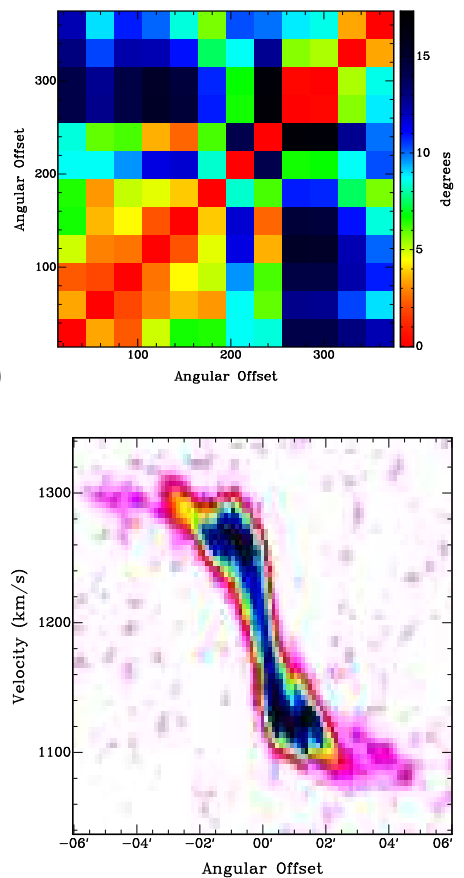

Figure 3: UGC 3580, contours in a) $1,4,7,10,13,17 \mathrm{M}_{\odot} / \mathrm{pc}^{2}$, in d) $0.5,1,4,7,10,13,17 \mathrm{M}_{\odot} / \mathrm{pc}^{2}$ 
a)
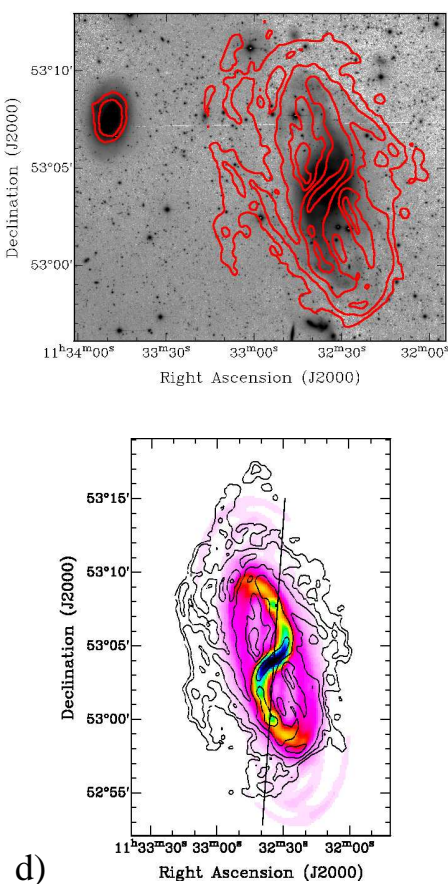

b)

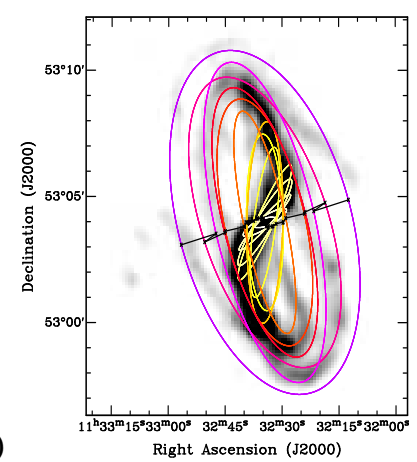

c)

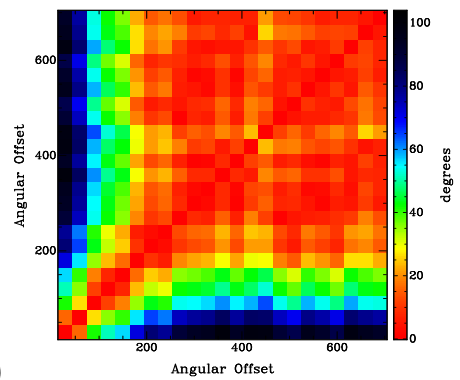

e)

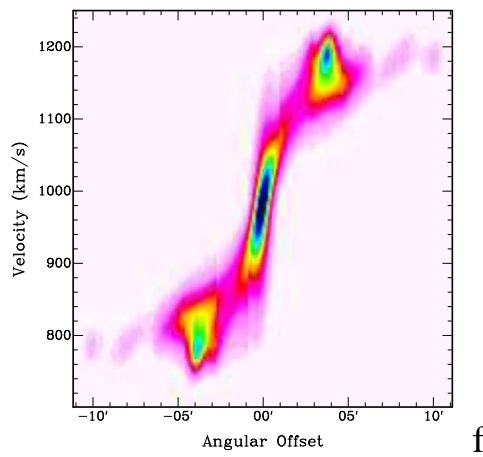

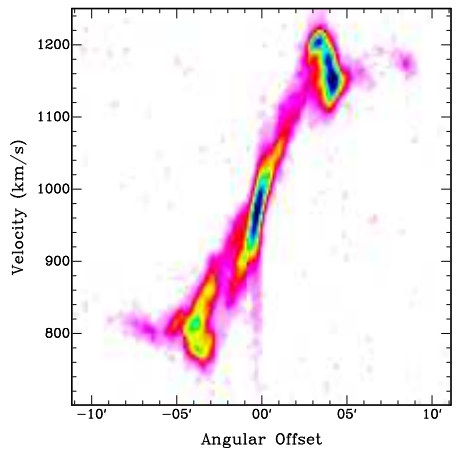

Figure 4: NGC 3718, in a) to the NE NGC 3729, contours in a) $0.7,2.1,6.3,18.9 \mathrm{M}_{\odot} / \mathrm{pc}^{2}$, in d) $0.2,0.6,1.8,5.4,16.2 \mathrm{M}_{\odot} / \mathrm{pc}^{2}$

a)

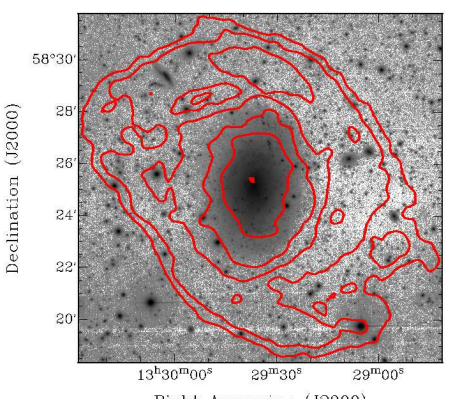

Right Ascension (J2000)

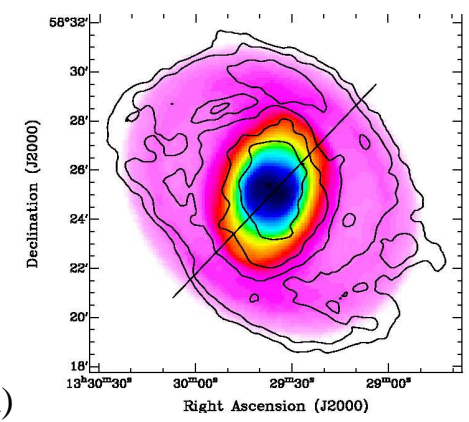

b)

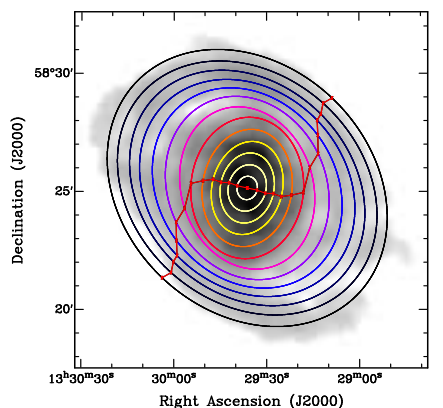

c)
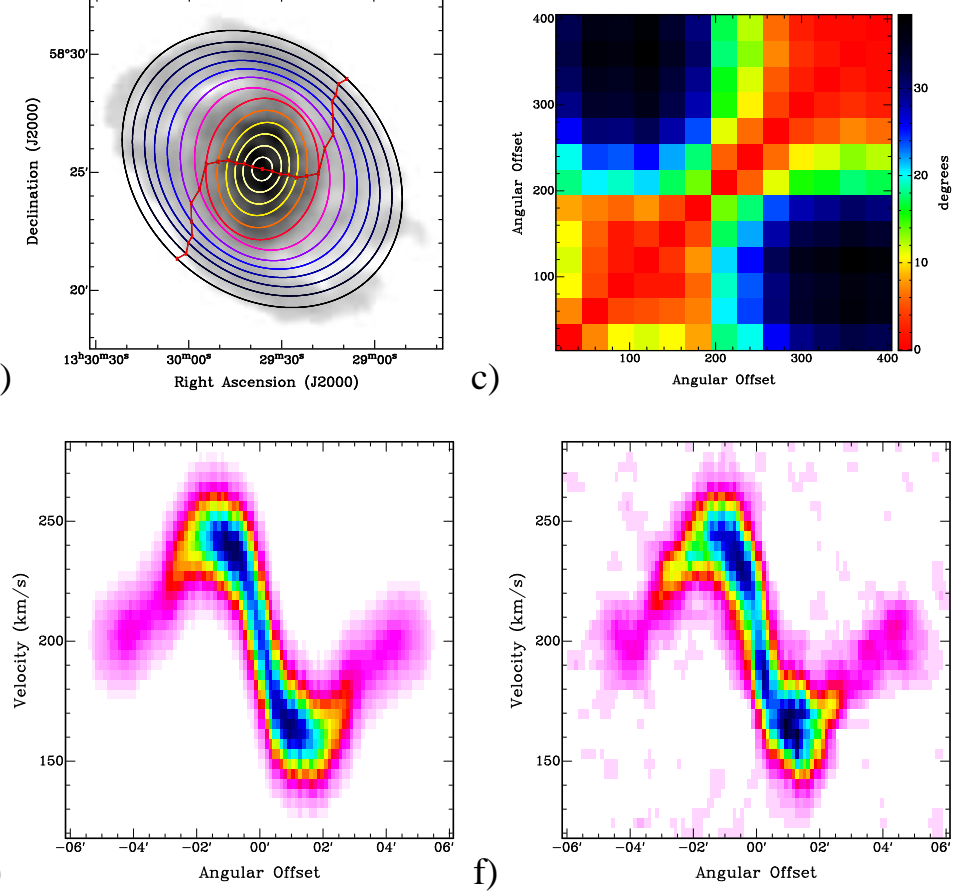

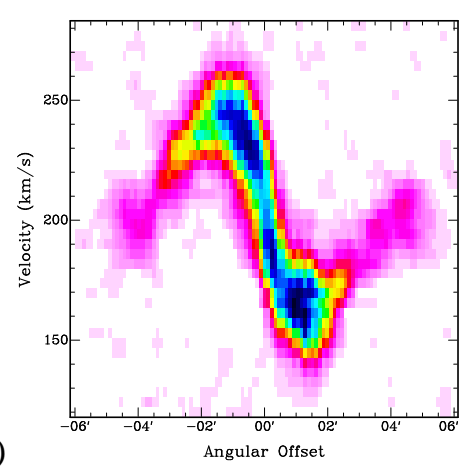

Figure 5: NGC 5204, contours in a) and d) 0.6, 1.2, 2.4,4.8,9.2, 18.4 $\mathrm{M}_{\odot} / \mathrm{pc}^{2}$ 


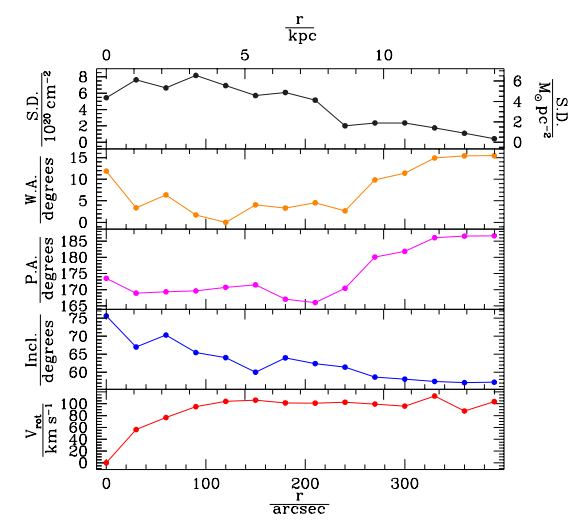

a) $\stackrel{r}{\mathrm{P}}$

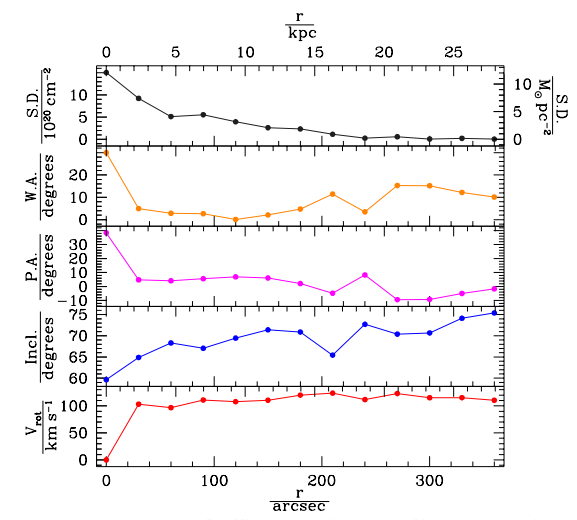

S.D.: HI surface density
c) W.A: warp angle
P. radius
P.A. position angle Incl.: inclination

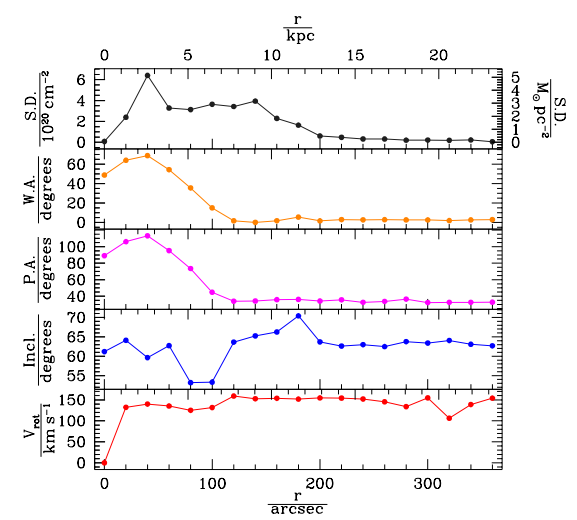

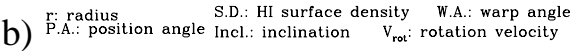

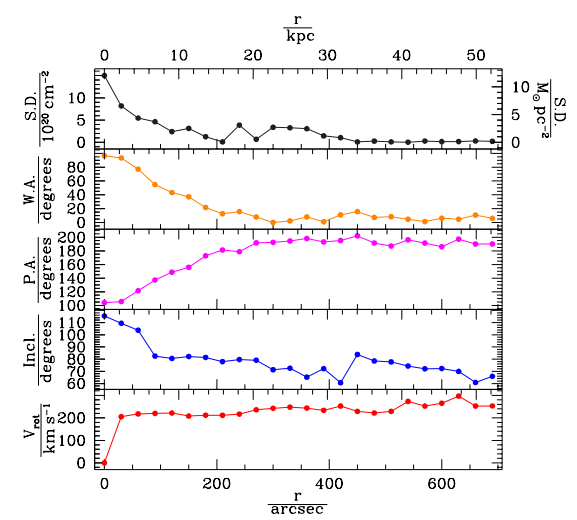

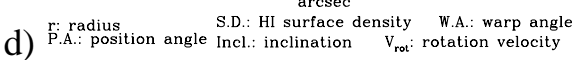

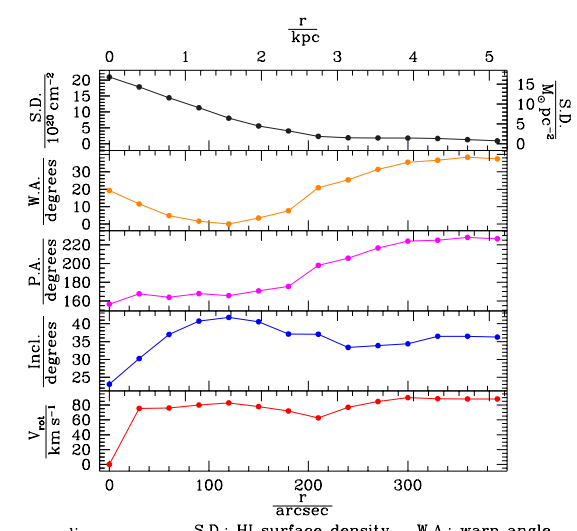

e) $\begin{aligned} & \text { r: radius } \\ & \text { P.A. position angle S.D.: HI surface density }\end{aligned}$

Figure 6: Parameters of our best-fit models, from top to bottom in each diagram: HI surface-density, warp angle, position angle, inclination, and rotation velocity. a) NGC 2541, b) NGC 2685, c) UGC 3580, d) NGC 3718, e) NGC 5204 